Research Article

\title{
The Influence of Depression and Anxiety on Neurological Disability in Multiple Sclerosis Patients
}

\author{
Vitalie Văcăraș, ${ }^{1,2}$ Veronica Văcăraș, ${ }^{3}$ Cristina Nistor $\left(\mathbb{D},{ }^{1}\right.$ Daniela Văcăraș, ${ }^{2}$ \\ Adrian Nicolae Opre, ${ }^{4}$ Petronela Blaga, ${ }^{3}$ and Dafin F. Mureșanu ${ }^{1,2}$ \\ ${ }^{1}$ Neurology Department, Cluj Emergency County Hospital, Cluj-Napoca, Romania \\ ${ }^{2}$ Department of Neurosciences, University of Medicine and Pharmacy, Cluj-Napoca, Romania \\ ${ }^{3}$ Civil Professional Psychotherapy Society, Cluj-Napoca, Romania \\ ${ }^{4}$ Psychology Department, Faculty of Psychology and Educational Sciences, Cluj-Napoca, Romania
}

Correspondence should be addressed to Cristina Nistor; cristinapinzaru@yahoo.com

Received 4 May 2020; Revised 15 June 2020; Accepted 24 June 2020; Published 3 July 2020

Academic Editor: Luigi Trojano

Copyright ( 2020 Vitalie Văcăraș et al. This is an open access article distributed under the Creative Commons Attribution License, which permits unrestricted use, distribution, and reproduction in any medium, provided the original work is properly cited.

Multiple sclerosis (MS) is a demyelinating disease of the central nervous system (CNS), affecting mostly young-aged people. As a chronic incurable disease, in most cases, it can lead to progressive neurological impairment and severe disability. Depression and anxiety are major distress factors for MS patients, being considerably aggravating elements for their functional capacity. In this study, we analysed the mood disorder distribution and the possible correlations between depression, anxiety, automatic negative thoughts, and MS disability. We took into consideration 146 MS patients, who completed a series of questionnaires: Beck Depression Inventory II (BDI-II), Endler Multidimensional Anxiety Scales-State (EMAS-S), and Automatic Thoughts Questionnaire (ATQ). The Expanded Disability Status Scale (EDSS) was used to measure the neurological disability. Of all patients, $30.1 \%$ had symptoms for depression and $11 \%$ presented suicidal thoughts. After analysing the correlation index between each variable, we found that there is a mild positive correlation between depression and the EDSS score and between anxiety and the EDSS score. A difference is found in the test scores according to the type of the MS disease. Also, automatic negative thoughts are strongly correlated with depression and anxiety, but do not mediate the path between psychological comorbidities and neurological impairment. Sociodemographic features and interferon-beta treatment were not related to the intensity of the mood disorders. The study suggests that depression and anxiety are frequently encountered among MS patients and these mental disfunctions have an impact on their disability. A proper identification of these risk factors may improve the quality of life for these patients.

\section{Introduction}

Multiple sclerosis (MS) is a chronic autoimmune inflammatory disease affecting the central nervous system (CNS) through demyelinating and degenerative lesions [1]. It is the most common chronic disabling disease of the CNS in young adults, affecting 2.3 million people worldwide. It is two times more common in females than in males, and its onset is usually at a young age, namely around 30 [2]. About half of these patients will develop a severe disability within 15 years of being diagnosticated [1].
There are four types of MS: clinically isolated syndrome (CIS), relapsing-remitting MS (RRMS), primary progressive MS (PPMS), and secondary progressive MS (SPMS). CIS is considered to be the first phase of the disease with symptoms explained by the demyelination and inflammatory lesions in CNS, with a duration of at least 24 hours, but not meeting the criteria for MS. Most of the patients present with RRMS, which is defined by multiple attacks of new or exacerbated neurological symptoms, followed by remission (partial or complete recovery) with no continuous progression of the disease during the recovery periods. Most of the cases of 
RRMS will progress over time into SPMS cases, with a gradual disability accumulation. To only $10 \%$ of patients, this progressive course of the disease may appear from the beginning, and these cases are categorized as having the PPMS form of the disease [3].

MS symptoms are variable, according to which area of the CNS is affected by demyelination. These include motor deficits with consecutive gait disturbance, visual acuity loss, fatigue, loss of coordination, sensory alterations (paresthesia, hypoesthesia), and sphincter incontinence, followed by anxiety, depression, and cognitive problems [2].

Studies found that MS can increase the risk for associated mental disorders. Depression and anxiety are the major distress factors, which impact the functionality and the quality of life for these patients [4]. In most cases, anxiety is associated with depression, thus having a higher symptom severity and a worsening functional capacity [5]. Identifying these comorbidities at the beginning is mandatory to improve their life quality and disability.

Depression is a mental disorder characterized by the loss of interest of previously enjoyable activities, accompanied by the incapacity to perform normal daily activities [6]. The risk for developing depression in the general population varies among gender, $5.1 \%$ for females and $3.6 \%$ for males, mostly affecting young-aged people from 18 to 45 years old. One factor to increase the risk of this pathology includes the presence of a chronic illness that offers the perception of health problems [4].

The prevalence of depression among MS patients is up to $50 \%$ of cases, a number 2-3 times higher than that of the general population [7]. Depression can occur in any moment of the disease, but with a higher risk in the first years after the diagnosis, even in the less severe forms of the disease. The patients experience fatigue, memory and concentration disturbances, insomnia, irritability, and loss of appetite, which are symptoms which may overlap with MS, leading to a difficult diagnosis [2].

Anxiety disorders are defined as a nonadaptative behavior which interferes with the normal function of the person, according to the Diagnostic and Statistical Manual of Mental Disorders (DSM-5) [8]. They are the most frequent among mental disturbances [9]. The prevalence of these disorders in MS patients is estimated to be $31 \%$, three times higher than in the general population. In MS patients, the most common are generalised anxiety disorder (18.6\%), panic disorder (10\%), and obsessive-compulsive disorder $(8.6 \%)$ [2].

Patients with MS present high levels of negative emotions and low levels of positive thoughts. Anxiety is a strong factor for depression development, in direct and indirect pathways. Therefore, anxiety should be evaluated initially in order to prevent or diagnose depression symptoms [10].

For MS patients, organic changes were associated with a higher depression risk, such as prefrontal cortex, anterior temporal lobe, parietal lobe, and left arcuate fasciculus. Also, cortical atrophy of frontal lobes, hippocampus variations, and neuroendocrine mechanisms are involved in mood disorders. Patients with MS have a hyperactivity of the hypothalamus-pituitary-adrenal axis, leading to normal morning and elevated evening levels of cortisol [2]. There are some psychological factors found in MS patients that are linked to depression. Mechanisms such as escape-avoidance (daydreaming for example) and emotional respite were associated with depressed people, while cognitive reframing (the attempt to obtain new perspectives) was related to a lower depression prevalence [1]. In MS patients, the inflammatory process leads to a destructive process in the serotoninergic system. Therefore, the functional reserve of serotonin seems to be reduced for these patients, demonstrated by low levels of humoral serotonin. In some life situations, these changes can precipitate anxiety and depression $[11,12]$.

The treatment for depression in MS is not different from the general population and should also include the use of adaptative coping mechanisms and cognitive-behavior therapy [13].

Disease-modifying therapies (DMTs) are medications aimed at reducing disability in time and MS activity. They influence the pathological process of the disease thorough different mechanisms of action [14]. Interferon-beta (IFNbeta) is a type of treatment which influences MS progression, currently contraindicated in individuals with severe depression, due to its uncertainty regarding the implication in this comorbidity [15]. IFN-beta was associated with a higher risk of depression in some studies [16]. However, there is no connection with the serotonin metabolism in IFN-beta-treated patients [12]. In recent studies, there was no relationship between IFN-beta and emotional disturbances [15]. Some authors suggest that depression is most likely to occur in an IFN-beta-treated individual only with a past history of depressive disorders. The depressive episode is more frequent in the first 6 months of treatment [11]. A specific causality remains controversial.

The Expanded Disability Status Scale (EDSS) is a standardized measure of disability in MS, used to evaluate at present and in dynamics the functional status of the patient. The neurological examination grades the disability of each system (pyramidal, brainstem, cerebellar, mental, sphincter, sensory, and visual) and offers a final score, ranging from 0 to 10 . Score 0 means normal neurological examination, 5 refers to a loss of ambulatory function and daily activity impairment, and 10 is death caused by MS complications [10].

Being considered an incurable disease which leads to major disability in most cases, neuropsychiatric complications are frequent in MS patients [2]. Psychiatric symptoms of anxiety, depression, and negative thoughts are concomitant pathologies for MS patients, which are found to be associated with an increased neurological disability measured by the EDSS score [17].

The objective of our research is to determine the correlation between depression, anxiety, automatic thoughts, and disability, based on our clinical experience.

\section{Material and Methods}

2.1. Participants and Procedure. The participants of this study were selected among the patients taken into evidence with multiple sclerosis at the Neurology Department in the Emergency Hospital Cluj-Napoca. The ethical and clinical 
TABLE 1: Baseline patients' characteristics.

\begin{tabular}{|c|c|}
\hline Characteristics & Study population $(n=146)$ \\
\hline \multicolumn{2}{|l|}{ Gender, $n(\%)$} \\
\hline Male & $41(28.08)$ \\
\hline Female & $105(71.92)$ \\
\hline \multicolumn{2}{|l|}{ Age } \\
\hline Median (SD) (y) & $40.8(11.5)$ \\
\hline $19-29 \mathrm{y}, n(\%)$ & $31(21.23)$ \\
\hline $30-39 y, n(\%)$ & $34(23.28)$ \\
\hline $40-49 \mathrm{y}, n(\%)$ & 47 (32.19) \\
\hline $50-70 y, n(\%)$ & $34(23.28)$ \\
\hline \multicolumn{2}{|l|}{ Living area, $n(\%)$} \\
\hline Rural & $33(22.6)$ \\
\hline Urban & $113(77.39)$ \\
\hline \multicolumn{2}{|l|}{ Education, $n(\%)$} \\
\hline $\mathrm{PhD}$, license, master & $60(44.5)$ \\
\hline High school, middle school & $81(55.4)$ \\
\hline \multicolumn{2}{|l|}{ Actual marital status, $n(\%)$} \\
\hline Married & $87(59.58)$ \\
\hline Not married & $59(40.42)$ \\
\hline \multicolumn{2}{|l|}{ MS type, $n(\%)$} \\
\hline RRMS & $128(87.67)$ \\
\hline SPMS & $10(6.84)$ \\
\hline CIS & $8(5.47)$ \\
\hline PPMS* & $0(0)$ \\
\hline \multicolumn{2}{|l|}{ EDSS score } \\
\hline Mean (range) & $2.3(0-6.5)$ \\
\hline $0-1.5, n(\%)$ & $48(32.87)$ \\
\hline $2-2.5, n(\%)$ & $43(29.45)$ \\
\hline $3-3.5, n(\%)$ & $38(26.02)$ \\
\hline $4-4.5, n(\%)$ & $6(4.1)$ \\
\hline $5-5.5, n(\%)$ & $7(4.79)$ \\
\hline $6-6.5, n(\%)$ & $4(2.73)$ \\
\hline \multicolumn{2}{|l|}{ Confirmed disease duration } \\
\hline Mean (SD) (y) & $6(6.32)$ \\
\hline$<1 \mathrm{y}, n(\%)$ & $19(13.01)$ \\
\hline $1-2 \mathrm{y}, n(\%)$ & $35(23.97)$ \\
\hline $3-9 y, n(\%)$ & $61(41.78)$ \\
\hline$\geq 10 \mathrm{y}, n(\%)$ & $31(21.23)$ \\
\hline \multicolumn{2}{|l|}{ Disease duration until diagnosis } \\
\hline Mean (SD) (y) & $2(4.9)$ \\
\hline
\end{tabular}

Abbreviations: $n$ : number of patients; SD: standard deviation; y: years; RRMS: relapsing-remitting MS; SPMS: secondary progressive MS; CIS: clinically isolated syndrome; PPMS: primary progressive MS. ${ }^{*}$ Limited number of PPMS patients who visited the clinic in the collecting data period did not agree to participate in this study.

authorizations were obtained. Each patient who agreed to be included in the study signed an informed consent and completed a series of standardized questionnaires: Beck Depression Inventory-II (BDI-II) for depression evaluation, Endler Multidimensional Anxiety Scales-State (EMAS-S) for anxi-
TABLE 2: Treatment details for studied patients.

\begin{tabular}{lcc}
\hline Actual DMT & $\begin{array}{c}\text { Number of patients } \\
(\%), \text { mean years of } \\
\text { treatment }\end{array}$ & $\begin{array}{c}\text { Number of patients with } \\
\text { previous treatment } \\
\text { (mean number of years) }\end{array}$ \\
\hline $\begin{array}{l}\text { IFN-beta-1a i.m. } \\
\text { IFN-beta-1a s.c. }\end{array}$ & $21(31.51), 2.5$ & $6(3.5)$ \\
Glatiramer & $45(30.82), 4.9$ & $6(1.5)$ \\
acetate & $9(6.16), 3.2$ & $2(6)$ \\
Natalizumab & $4(2.74), 7.3$ & $1(1)$ \\
IFN-beta-1b & $2(1.37), 1.5$ & $7(5.4)$ \\
Mitoxantrone & $1(0.68), 9.0$ & 0 \\
$\begin{array}{l}\text { Dimethyl } \\
\text { fumarate }\end{array}$ & $18(12.33), 0.0$ & 0 \\
No medication & . & 125 \\
\hline
\end{tabular}

Abbreviations: IFN: interferon; i.m.: intramuscular; s.c.: subcutaneous.

ety, and Automatic Thoughts Questionnaire (ATQ) for automatic thought interpretation, as well as a sociodemographic questionnaire created to have a data profile, including age, social status, educational degree, and religion beliefs. The neurologist evaluated the Expanded Disability Status Scale (EDSS).

The inclusion criteria were MS adult patients with an established MS diagnosis, who accepted to complete all questionnaires in a period of five months when data was collected. We excluded the patients who did not integrally complete the questionnaires and those with an incomplete neurological examination or medical history.

In total, we included 146 MS patients, 41 males and 105 females, with a 40.8-year-old median age. The main baseline characteristics of the patients, including sociodemographical factors and details regarding MS evolution, are presented in Table 1. The majority of the patients from our study were treated with different forms of interferon-beta (IFN-beta), but other treatments were also used (Table 2).

2.2. Instruments. Beck Depression Inventory-II (BDI-II) is a measurement of clinical and subclinical depression, using 21 statements, such as depressive mood, pessimism, the feeling of failure, the lack of pleasure, the sense of guilt, punishment, self-unsatisfaction, suicidal ideas, crying, irritability, social isolation, fatigue, body image disturbances, and insomnia. The final score establishes the severity of depression: 14-19 points for mild depression, 20-28 points for moderate depression, more than 29 points for severe depression [18].

Endler Multidimensional Anxiety Scales-State (EMAS-S) are three scales used to evaluate different anxiety components: anxiety as a mood, the cognitive part of anxiety, and the total anxiety level [19].

Automatic Thoughts Questionnaire (ATQ) is a questionnaire for measurement of the occurrence of automatic negative thoughts, negative self-statements, associated with depression. It is composed of 15 items in which the patient states the frequency of the irrational thoughts from 1 to 5 , in the past month [20]. The result is divided into 5 categories. In the first one, we find an extreme low level of dysfunctional 
thoughts, in the second class there are low levels, in the third category we have a medium level, class 4 represents a high frequency of automatic negative thoughts, and the fifth class is in very high frequencies.

The Expanded Disability Status Scale (EDSS) was calculated by the neurologist according to the neurological examination. The results were outlined according to the neurological status of the patients at the moment of examination: 0 points (p.) for a complete normal neurological examination, 1-1.5 p. for pathological signs but no deficits, 2-2.5 p. for minimal disability, 3-3.5 p. in case of moderate disability, 4-4.5 p. for severe disability, 5-5.5 p. in case of gait impairment but possible for less than $200 \mathrm{~m}, 6-6.5 \mathrm{p}$. for a permanent dependence for walking, 7-7.5 p. for a patient immobilised in a chair, 8-8.5 p. for a dependent to bed patient but still able to perform some self-care tasks, and 9-9.5 p. for a completely immobilised and dependent patient [21].

\section{Results}

After we collected the information from all complete questionnaires, neurological examination, and medical history, the outcome was that $30.1 \%$ (44 patients) of our study participants have a form of depressive disorder, defined as a BDI score higher than 14 points, of which $14 \%$ have mild depression (BDI score $14-19$ points), $11 \%$ medium (BDI score 2028 points), and $5 \%$ a severe form (higher than 29 points) (Figure 1).

We summarize the test results in Table 3. The higher scores of all tests were obtained for patients treated with mitoxantrone, followed by glatiramer acetate and IFN-beta1b. Almost $72 \%$ of patients complain of an important level of fatigue.

In the first two years of MS diagnosis, the mean BDI score was 8.31 points, while for those with a disease duration longer than 2 years, the mean score is 11.82 points.

In search for any statistical differences $(p<0.05)$ between males and females regarding all three test scores, we calculated a $p$ value of 0.42 for the relationship with depression, 0.18 for anxiety, and 1 for automatic thoughts, with all $p$ values indicating that in our group, test results were not influenced by gender in a significant matter.

Regarding the MS type, we observe a tendency of higher values for mean scores in the BDI-II and EMAS-S tests with the worsening of the disease prognosis, the distribution of the mean scores being stated in the two histograms in Figures 2 and 3.

We calculated the possible correlation between each psychological test and the EDSS score, and we centralised the information in Table 4.

According to our data analysis, there is a low degree, mild positive correlation between depression and neurological disability in MS, with a correlation coefficient $r=0.22$ for the BDI-II and EDSS scales. We also discovered a mild positive correlation between the anxiety and disability scales, with a coefficient of 0.22 for EMAS-S and EDSS scores. The finding of an almost null correlation index between EDSS and the ATQ test suggests that there is no statistically significant connection between automatic thoughts and the severity of the

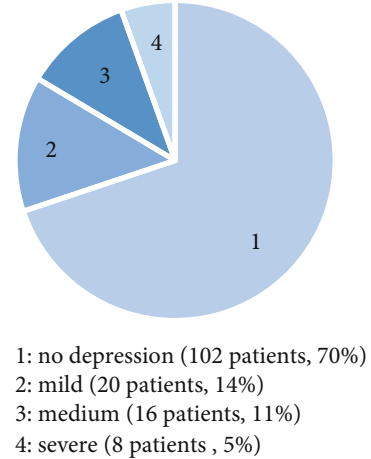

Figure 1: Depression type according to the BDI-II scale.

disease. However, with an $r$ of 0.65 for ATQ and BDI-II scores, there is a moderate to high relationship between automatic thoughts and depression. Moderate correlations were also found for BDI-II and EMAS-S $(r=0.52)$ and for ATQ and EMAS-S $(r=0.54)$.

While calculating the total EDSS score, we also took into consideration each functional system (FS) parameter, which allows us to differentiate the main symptoms or signs of the disease. The seven systems included in the EDSS scale that we evaluated are coded as follows: A: pyramidal system, B: cerebellar, C: brainstem, D: sensory, E: bladder and bowel, F: visual, and G: mental or cerebral function. The mean score for each FS is presented in Figure 4.

We calculated the correlation for each variable from the EDSS score with the three psychological tests. The results are summarized in Table 5, which suggests a mild connection between pyramidal symptoms and higher depressive elements, also between brainstem disturbances and anxiety disorder; the other results have shown no statistical significance.

In our analysis, we also included the hypothesis that the actual IFN treatment may have an influence on mood disorders. We used the cut-off value of $p<0.05$ to obtain statistical significance. We defined depression as a BDI-II score higher than 14 points, important anxiety as an EMAS-S score higher than 50 points, and the presence of important negative thoughts as an ATQ value over 45 points. Thus, with a $p$ value of 0.27 , we found no statistically significant difference between the presence of IFN treatment for depressive symptoms. Also, there is no influence of IFN on anxiety scores $(p=1)$ or on the presence of negative thoughts $(p=0.58)$. If we compared the group with any type of current DMT treatment with the one with no specific medication, there is also no influence regarding the depression score on the BDI-II test $(p=1)$. The same result was obtained for the group with previous DMT; there is no influence on depressive symptoms regarding the absence or presence of any treatment in the past $(p=0.29)$.

Almost $11 \%$ of our patients presented different types of suicidal thoughts, identified by a score higher than 1 in specific questions in the BDI-II scale. The mean score for suicidal ideation was 1.27 points for females and 1 point for males. Married individuals had a mean of 1.14 points and unmarried of 1.22 points. 
TABLe 3: Psychological test score details according to MS type and treatment.

\begin{tabular}{|c|c|c|c|}
\hline & BDI & ATQ & EMAS-S \\
\hline \multicolumn{4}{|l|}{ MS type, mean } \\
\hline CIS & 5.87 & 27.37 & 25.62 \\
\hline RRMS & 10.34 & 27.35 & 35.21 \\
\hline SPMS & 16.5 & 29.2 & 43.4 \\
\hline \multicolumn{4}{|l|}{ Actual DMT, mean } \\
\hline IFN-beta-1a i.m. & 10.20 & 26.33 & 34.63 \\
\hline IFN-beta-1b & 11.75 & 29.50 & 41.75 \\
\hline Glatiramer acetate & 12.62 & 29.89 & 35.16 \\
\hline Mitoxantrone & 13.50 & 33.50 & 50.00 \\
\hline IFN-beta-1a s.c. & 6.90 & 27.29 & 30.95 \\
\hline Natalizumab & 8.22 & 20.33 & 34.56 \\
\hline No current medication & 10.72 & 27.44 & 38.67 \\
\hline Total $(n=146)$, mean $[S D]$ (range) & $10.52[9.67](0-54)$ & $27.48[11.23](15-59)$ & $35.25[16.4](20-87)$ \\
\hline
\end{tabular}

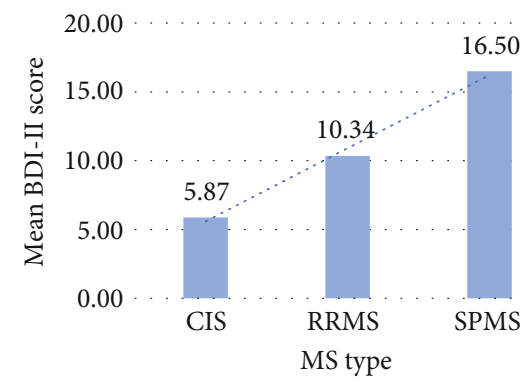

FIGURE 2: Depression score according to MS type.

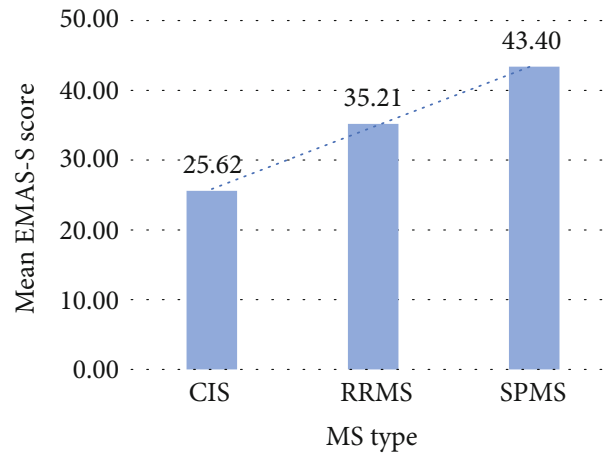

Figure 3: Anxiety score according to MS type.

TABLE 4: The correlation matrix for tested variables.

\begin{tabular}{lccc}
\hline & EDSS & BDI-II & ATQ \\
\hline EDSS & - & & \\
BDI-II & $0.22^{*}$ & - & \\
ATQ & 0.06 & $0.65^{*}$ & - \\
EMAS-S & $0.22^{*}$ & $0.52^{*}$ & $0.54^{*}$ \\
\hline
\end{tabular}

*Bidirectional testing with positive statistical significative correlation at $p<$ $0.01, n=146$

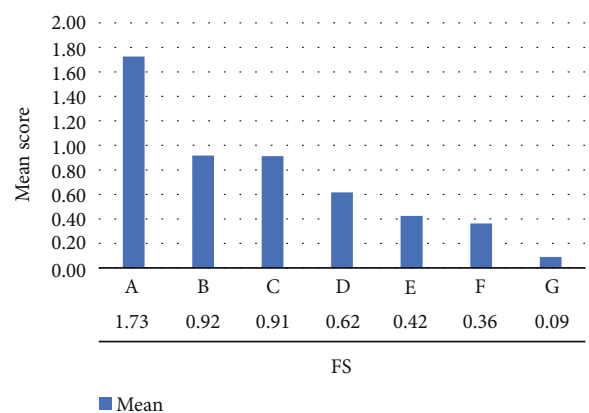

Figure 4: Mean EDSS score for every FS.

TABLE 5: Correlations between every FS of EDSS and psychological tests.

\begin{tabular}{lccc}
\hline FS & BDI-II & ATQ & EMAS-S \\
\hline A & 0.30 & 0.10 & 0.16 \\
B & 0.18 & 0.06 & 0.20 \\
C & 0.21 & 0.08 & 0.33 \\
D & 0.21 & 0.06 & 0.19 \\
E & 0.16 & 0.07 & 0.15 \\
F & -0.06 & -0.06 & -0.03 \\
G & 0.24 & 0.15 & 0.20 \\
\hline
\end{tabular}

\section{Discussion}

MS is a chronic disease which is considered incurable, leading in most cases to neurological impairment and physical and psychical disabilities. MS creates a major distress for the patients, depression and anxiety being the most severe psychological comorbidities [10]. Depression occurred in up to $50 \%$ of people diagnosed with MS [1], with a variation according to the population tested and the scales used as diagnosis, but the most frequent percentage found in literature is around $30 \%$. A study accomplished on more than 115,000 patients outlines that $25.7 \%$ of MS cases were also diagnosed with a form of depression [22]. Our results from 
the Neurology Department in the Emergency Hospital ClujNapoca revealed similar results with the ones from the literature, $30.1 \%$ of MS patients from our clinic presented a depressive disorder.

Some scientific papers state that depression is more frequent in the first years after diagnosis [10], but our mean BDI-II score for less or more than 2 years from diagnosis revealed even the opposite, with greater depressive symptoms in the group with a long-term disease (11.8 points vs. 8.3 points). This difference might be due to the inequality between the two groups.

Depression should be evaluated at the beginning of the disease and also during the course of the pathology, due to a decreased life quality that can be improved with therapy or due to a large prevalence of suicide intention [23]. The suicidal rate in MS patients is at least twice larger than in the general population [24], with suicidal attempts for a quarter of these patients [25]. Up to $15 \%$ of all MS deaths may be related to a suicidal fact, almost half of them appearing in the first 5 years after diagnosis. The risk for suicide is associated with the severity of the illness, depression level, quality of life, and being unmarried and is more frequent in males [2]. In our analysis, $11 \%$ of patients declared suicidal thoughts in the BDI-II test, but there is no major difference between gender and marital status regarding the suicide ideation. Both males and females, married or unmarried, expressed the same predisposition for having suicidal thoughts. This may be due to a moderate number of individuals included, which cannot provide proper statistical information.

Anxiety is largely found in MS patients, as studies indicate [17]. The results in our data analysis from the anxiety questionnaire show that MS patients have a very high level of anxiety, with a mean EMAS-S score of 35.25.

Although depression and anxiety are generally more frequent in the female population [4], our test results found no difference between genders regarding the scores for all three tests.

There are important differences for mean scores in the BDI-II and EMAS-S tests for each MS type from our group, which suggest that the type of the disease may influence the mood disorders. Thus, for CIS patients, who also have the mild disease course compared to others, the mean score for depression is only half of the score for RRMS patients. Also, SPMS has a much higher value than the other two categories, probably due to the severity of the disease which leads to a progressive neurological impairment. The same tendency is found for the anxiety scale, where the lowest scores are predominantly for CIS cases and the most important anxiety for SPMS individuals.

Some studies suggest that a higher EDSS score is associated with the presence of a mood disorder, including depression and anxiety [17]. This may be due to the fact that neurological impairment in general might affect the quality of life and predispose to greater depression symptoms, or the severity of the disease itself can lead to greater anxiety. In another large study based on thousands of MS cases, it was also sustained that the presence of depression was linked to the severity of the EDSS score, which implies that MS individuals with depression were predisposed to a worse disability [26].

The hypothesis that depression and anxiety are connected to the neurological disability was only partially confirmed, as the correlation index for EDSS and BDI-II and for EDSS and EMAS-S scores was in both cases 0.22 , which suggests only a low degree positive correlation. Our results are similar to the ones in another study where there was a low impact of the functional status on depression [10].

Although our study results outline that automatic negative thoughts have an influence on depression and anxiety, they do not mediate the relationship between depression or anxiety and the disability scale. Some possible explanations for this result may be that in the analysed population, there are other important mediators, such as irrational cognitive thoughts, control, unconditioned self-acceptance, or the validity of the ATQ questionnaire on this specific statement.

Outcome measured for different DMTs outlines only a few differences regarding several well-being components, including depression [27]. There is a controversy regarding the precise relationship between IFN-beta treatment and depressive disorders, because it was initially associated with an increased risk for major depression episodes, especially in the first period of treatment [16]. However, recent studies suggest that these events are likely to occur for an already predisposed patient, with a past history of psychiatric comorbidity [11]. In our analysis, we found no statistically significant association between the IFN-beta treatment and a major score for both depression and anxiety or even for the presence of automatic negative thoughts. Also, by comparing the group of patients treated with any kind of DMT and the one with no specific medication at that time, there was also no difference on psychological test scores. Our results outline that the presence of mood disorders was not influenced by treatment.

From 146 patients included in our study, $72 \%$ had scores between 1 and 3 for fatigue in the BDI-II test. Fatigue is a symptom present in more than three quarters of MS patients but is an overlap symptom; there is still no consensus regarding the provenience of these symptoms which may be either due to multiple sclerosis or due to an underlying psychiatric condition [1].

In further analysis, we might take into consideration other possible mediators between the psychiatric condition and the neurological disability. A part of the data used for this study is based on a master dissertation [28].

\section{Conclusions}

MS is a widespread disease around the world that affects predominantly young people, leading in most cases to progressive neurological disability. Due to the major distress caused by the pathology, depression and anxiety are common comorbidities among these patients.

Our study reveals that there is a mild positive correlation between anxiety, depression, and neurological disability, which suggests that the psychiatric conditions contribute to the progression of the disease but not in an important manner. Interferon-beta treatment or other sociodemographical 
factors seem to have an insignificant contribution. From our clinical experience, $30.1 \%$ of patients present with depression symptoms, and $11 \%$ have suicidal thoughts.

These psychological statements should be evaluated initially and periodically for each MS patient, in order to diagnose psychiatric comorbidities on time, to reduce the patients' disability and to improve their life quality.

\section{Data Availability}

The data that we used as the basis of our manuscript are protected by the personal data privacy and the inform consent signed by each participant of the study. However, if it is necessary, the corresponding author can provide it upon request for the researchers who meet the criteria for access to confidential data.

\section{Conflicts of Interest}

The authors declare no conflicts of interest.

\section{References}

[1] S. B. Patten, R. A. Marrie, and M. G. Carta, "Depression in multiple sclerosis," International Review of Psychiatry, vol. 29, no. 5, pp. 463-472, 2017.

[2] C. Silveira, R. Guedes, D. Maia, R. Curral, and R. Coelho, "Neuropsychiatric Symptoms of Multiple Sclerosis: State of the Art," Psychiatry Investigation, vol. 16, no. 12, pp. 877888, 2019.

[3] P. Browne, D. Chandraratna, C. Angood et al., "Atlas of Multiple Sclerosis 2013: A growing global problem with widespread inequity," Neurology, vol. 83, no. 11, pp. 1022-1024, 2014.

[4] H. A. Razzak, A. Harbi, and S. Ahli, "Depression: Prevalence and Associated Risk Factors in the United Arab Emirates," Oman Medical Journal, vol. 34, no. 4, pp. 274-282, 2019.

[5] K. H. Jones, D. V. Ford, P. A. Jones et al., "A Large-Scale Study of Anxiety and Depression in People with Multiple Sclerosis: A Survey via the Web Portal of the UK MS Register," PLoS ONE, vol. 7, no. 7, p. e41910, 2012.

[6] N. Fekadu, W. Shibeshi, and E. Engidawork, "Major Depressive Disorder: Pathophysiology and Clinical Management," Journal of Depression and Anxiety, vol. 6, no. 1, pp. 255-257, 2017.

[7] R. E. Boeschoten, A. M. J. Braamse, A. T. F. Beekman et al., "Prevalence of depression and anxiety in Multiple Sclerosis: A systematic review and meta-analysis," Journal of the Neurological Sciences, vol. 372, pp. 331-341, 2017.

[8] American Psychiatric Association, Diagnostic and Statistical Manual of Mental Disorders, American Psychiatric Association, Arlington, VA, 5th edition, 2013.

[9] R. C. Kessler, W. T. Chiu, O. Demler, K. R. Merikangas, and E. E. Walters, "Prevalence, severity, and comorbidity of 12month DSM-IV disorders in the national comorbidity survey replication," Archives of General Psychiatry, vol. 62, no. 6, pp. 617-627, 2005.

[10] M.-C. Gay, C. Bungener, S. Thomas et al., "Anxiety, emotional processing and depression in people with multiple sclerosis," BMC Neurology, vol. 17, no. 1, p. 43, 2017.
[11] L. A. Palé, J. L. Caballero, B. S. Buxareu, P. S. Serrano, and V. P. Solà, "Systematic review of depression in patients with multiple sclerosis and its relationship to interferon $\beta$ treatment," Multiple Sclerosis and Related Disorders, vol. 17, pp. 138-143, 2017.

[12] T. V. Baidina, T. N. Trushnikova, and M. A. Danilova, "Interferon-induced depression and peripheral blood serotonin in patients with multiple sclerosis," Zhurnal nevrologii i psikhiatrii im. S.S. Korsakova, vol. 118, no. 8, pp. 77-81, 2018.

[13] L. B. Grech, L. A. Kiropoulos, K. M. Kirby, E. Butler, M. Paine, and R. Hester, "Importance of Coping in the Relationship Between Executive Function and Quality of Life in People with Multiple Sclerosis," International Journal of MS Care, vol. 21, no. 5, pp. 201-206, 2019.

[14] D. Robertson and N. Moreo, "Disease-modifying therapies in multiple sclerosis: overview and treatment considerations," Federal Practitioner, vol. 33, no. 6, pp. 28-34, 2016.

[15] J.-C. Ouallet, F. Radat, A. Creange et al., "Evaluation of emotional disorders before and during treatment with interferon beta in patients with multiple sclerosis," Journal of the Neurological Sciences, vol. 413, no. 1, p. 116739, 2020.

[16] H. J. I. de Jong, E. Kingwell, A. Shirani et al., "Evaluating the safety of $\beta$-interferons in MS," Neurology, vol. 88, no. 24, pp. 2310-2320, 2017.

[17] K. A. McKay, H. Tremlett, J. D. Fisk et al., "Psychiatric comorbidity is associated with disability progression in multiple sclerosis," Neurology, vol. 90, no. 15, pp. e1316-e1323, 2018.

[18] A. T. Beck and R. A. Steer, Manual for the Beck Depression Inventory II, Psychological Corporation, San Antonio, TX, 1996.

[19] N. S. Endler, J. D. Parker, R. M. Bagby, and B. J. Cox, "Multidimensionality of state and trait anxiety: Factor structure of the Endler Multidimensional Anxiety Scales," Journal of Personality and Social Psychology, vol. 60, no. 6, pp. 919-926, 1991.

[20] S. D. Hollon and P. C. Kendall, "Cognitive self-statements in depression: development of an automatic thoughts questionnaire," Cognitive Therapy and Research, vol. 4, no. 4, pp. 383395,1980

[21] J. F. Kurtzke, "Rating neurologic impairment in multiple sclerosis: An expanded disability status scale (EDSS)," Neurology, vol. 33, no. 11, pp. 1444-1452, 1983.

[22] S. B. Patten, C. A. Beck, J. V. A. Williams, C. Barbui, and L. M. Metz, "Major depression in multiple sclerosis: a populationbased perspective," Neurology, vol. 61, no. 11, pp. 1524-1527, 2003.

[23] R. Kalb, A. Feinstein, A. Rohrig, L. Sankary, and A. Willis, "Depression and Suicidality in Multiple Sclerosis: Red Flags, Management Strategies, and Ethical Considerations," Current Neurology and Neuroscience Reports, vol. 19, no. 10, p. 77, 2019.

[24] H. Bronnum-Hansen, E. Stenager, N. Stenager, and N. KochHennksen, "Suicide among Danes with multiple sclerosis," Journal of Neurology, Neurosurgery \& Psychiatry, vol. 76, no. 10, pp. 1457-1459, 2005.

[25] R. A. Marrie, S. Reingold, J. Cohen et al., "The incidence and prevalence of psychiatric disorders in multiple sclerosis: A systematic review," Multiple Sclerosis Journal, vol. 21, no. 3, pp. 305-317, 2015.

[26] S. Binzer, K. A. McKay, P. Brenner, J. Hillert, and A. Manouchehrinia, "Disability worsening among persons 
with multiple sclerosis and depression," Neurology, vol. 93, no. 24, pp. e2216-e2223, 2019.

[27] B. I. Glanz, J. Zurawski, C. T. Gonzalez et al., "Comparison of health-related quality of life across treatment groups in individuals with multiple sclerosis," Multiple Sclerosis and Related Disorders, vol. 40, no. 2020, p. 101944.

[28] V. Văcăraș, “Disfuncții emoționale la pacienții cu scleroză multiplă (emotional disturbances in multiple sclerosis patients)," Unpublished Master's Dissertation, Psychology Department, Faculty of Psychology and Educational Sciences, Cluj-Napoca, Romania, 2017. 